ज्ञ FRANÇAISE

$\supset$ DE

때 PÉDAGOGIE

\section{Revue française de pédagogie}

Recherches en éducation

159 | avril-juin 2007

Politique et rhétoriques de l'« École juste » avant la Cinquième République

\title{
La Quatrième République et les questions de l'égalité et de la justice dans l'enseignement du second degré : le changement sans la réforme
}

The Fourth Republic and the problems of equality and fairness in secondary teaching: changing without reforms

La Cuarta República y las cuestiones de la igualdad y justicia en la enseñanza de segundo grado: el cambio sin la reforma

Die Vierte Republik und die Fragen der Gleichheit und der Gerechtigkeit in der

Sekundarstufe: Wandel ohne Reform

\section{André D. Robert}

\section{OpenEdition \\ Journals}

Édition électronique

URL : http://journals.openedition.org/rfp/1064

DOI : 10.4000/rfp.1064

ISSN : 2105-2913

Éditeur

ENS Éditions

Édition imprimée

Date de publication : 1 avril 2007

Pagination : 81-92

ISBN : 978-2-7342-1090-0

ISSN : 0556-7807

\section{Référence électronique}

André D. Robert, «La Quatrième République et les questions de l'égalité et de la justice dans

l'enseignement du second degré : le changement sans la réforme », Revue française de pédagogie [En ligne], 159 | avril-juin 2007, mis en ligne le 01 avril 2011, consulté le 03 mai 2019. URL : http:// journals.openedition.org/rfp/1064; DOI : 10.4000/rfp.1064 


\section{La Quatrième République et les questions de l'égalité et de la justice dans l'enseignement du second degré : le changement sans la réforme}

André D. Robert

Le présent article vise à montrer en quoi, aux lendemains de la seconde guerre mondiale, sous le régime de la Quatrième République, alors même qu'aucun des nombreux projets de réforme de l'école n'aboutit, s'opèrent néanmoins des changements réels (quantitatifs et qualitatifs, structurels et curriculaires) et se met en place une matrice intellectuelle abordant d'une manière en partie nouvelle les questions d'égalité et de justice scolaires. Tout en s'inscrivant dans une continuité avec le(s) projet(s) d'école unique, la réforme Jean Zay et un début de démocratisation du second degré avant guerre, cette matrice inscrit durablement à l'agenda politique la nécessité de transformer les accès au premier cycle ; elle offre toutefois une assez large variété de traitement de la question de l'égalité, qui, ne pouvant être abordée que sur le mode de la complexité, donne matière à des approches soit très progressistes et ambitieuses (plan Langevin-Wallon), soit en demi-teinte (démo-élitisme), soit de refus pur et simple, soit encore de moindre ambition mais pragmatiques (décisions de janvier 1959).

Descripteurs (TEE) : 1946-1958, changement social, égalité de chances, France, réforme de l'enseignement, système d'enseignement.

$\mathrm{N}^{\text {topan }}$ otre périodisation retient la Quatrième République comme son épicentre mais, on le sait, il n'y a jamais, en histoire, de commencement ni de coupure absolus, ce qui supposera de porter le regard quelque peu en deçà du 24 octobre 1946 et légèrement au-delà du 4 octobre 1958, dates respectives de promulgation des IV et $V^{e}$ Républiques. À propos de la question éducative qui nous occupe, il sera en effet nécessaire de se reporter, pour l'amont, non seulement à la Libération stricto sensu mais à des événements et décisions qui la préparent, tels que la création à Alger du Comité français de Libération nationale (CFLN) puis sa transformation en Gouvernement provisoire de la République française (GPRF) ; pour l'aval, à l'ordonnance et au décret du 6 janvier 1959 qui portent réforme de l'enseignement. Quant au cœur de cette question éducative, il touche principalement à la scolarisation dans le second degré, et mobilise quasi obligatoirement les concepts d'égalité et de justice, dont la compréhension n'apparaît cependant pas stabilisée, comme certains débats entre partisans de l'école unique ont pu le montrer 
depuis 1918 (Garnier, 2007 ; voir aussi Barreau \& Legrand, 1998 ; Garcia, 1994).

L'exposé des motifs du premier projet réformateur de la période est on ne peut plus direct dans la formulation du problème essentiel, comportant parmi ses sous-titres: “Le vrai problème; égalité réelle devant l'enseignement », et argumentant ainsi : « Les conciliations tentées dans le passé, les prétendues réformes [...] ont été inopérantes parce qu'elles ne tranchaient pas la vraie, la seule question, celle de l'égalité réelle devant l'enseignement [...] L'école unique, école secondaire pour tous faisant directement suite à l'école primaire pour tous en une progression continue, n'est pas encore réalisée » (1). À suivre ces appréciations, la solution aurait pu être simple dès lors qu'étaient réunies les conditions politiques de sa mise en œuvre comme c'était le cas avec les gouvernements d'union nationale issus de la Résistance : instituer un second temps de scolarité obligatoire identique pour tous. Cela aurait été se placer dans la perspective de ce que, en nous inspirant librement du philosophe américain Michaël Walzer (1997), nous proposons d'appeler l'égalité simple comme égale distribution à tous d'un bien quelconque, assurant son égale et réelle possession par tous. S'il est logique qu'un texte constitutionnel s'y réfère implicitement comme principe de droit - ainsi le préambule de la constitution du 17 octobre 1946, fondatrice de la IV République, stipulant que " La nation garantit l'égal accès de l'enfant et de l'adulte à l'instruction, à la formation professionnelle et à la culture »- une telle conception, rapportée à la réalité, s'avère difficilement accessible, à moins, souligne M. Walzer (1997) de recourir à un État extrêmement contraignant veillant à maintenir chacun strictement au même niveau II. C'est en fait à une notion d'égalité complexe que les experts et les décideurs, même les plus idéalistes, sont toujours confrontés dans leurs tentatives de concrétiser une réforme. Dans la mesure où il s'agit bien d'égalité complexe et non pas de concession aux inégalités au nom de ladite complexité, on doit en quelque sorte se représenter un curseur se déplaçant d'un point extrême à l'autre sur un segment, curseur exprimant selon sa position le degré de démocratisation atteint (sur cette notion, Isambert-Jamati, 1972) : à une extrémité, point de l'égalité simple et absolutisée, idéal inaccessible, à l'autre extrémité, point audelà duquel s'opère un basculement dans une inégalité elle-même manifeste (par exemple l'interdiction d'accès de certains enfants à tel type d'enseignement ou de filière) ; l'appréciation du meilleur endroit où positionner le curseur au nom d'une revendication d'égalité dépend bien sûr d'options politiques et idéologiques, mais aussi de l'estimation des possibles contenus dans le contexte et la conjoncture. On pourra en tout état de cause convenir avec M. Walzer, au titre des limites à respecter en vue d'éviter un basculement vers l'inégalité pure et simple, qu'«en dépit de l'existence de maintes petites inégalités le processus de conversion » d'un bien, en l'occurrence l'éducation, en un autre, par exemple le pouvoir ou l'aisance sociale, ne doit pas engendrer « la multiplication de l'inégalité »(Walzer, 1997).

Quelle que soit leur sensibilité profonde, tous les réformateurs de l'après seconde guerre mondiale sont nécessairement aux prises avec une problématique de ce que nous appelons ici égalité complexe parce qu'ils doivent prendre en compte les origines de l'institution scolaire et l'élitisme de son enseignement secondaire traditionnel, la structuration sociodémographique du pays (encore majoritairement rural) et les coûts induits par un projet de démocratisation généralisée (à un moment où les budgets devaient d'abord servir à relever matériellement la France de ses ruines). Plusieurs questions vives traversent la période, qui vont d'ailleurs continuer à interroger le système, y compris après les décisions réformatrices effectives de 1959 : question de la conception même de la scolarisation " de second degré » et du lieu où elle doit être commencée ; question de la durée de cette scolarisation secondaire pour tous (quelle durée d'un éventuel tronc commun ?) ; question de la différenciation interne à introduire, de la situation de celleci dans le temps, de sa nature et de son ampleur; question des maîtres qui doivent y enseigner (et comment), question se posant de façon aiguë pour la phase de cette scolarité qui fait immédiatement suite à l'école élémentaire.

Nous retenons ici dix des projets de réforme qui ont vu le jour entre 1944 et 1958, dont aucun ne fut appliqué (2), conduisant certains analystes à parler de «non décision» (Donegani \& Sadoun, 1976). Pour autant, le bilan de la IV République en matière scolaire, comme en d'autres domaines d'ailleurs (et contrairement à une idée reçue qu'ont contribué à répandre les fondateurs et les partisans de la Ve République) est loin d'être nul. Si l'analyse politique évoque aujourd'hui volontiers l'idée et l'expression de " changements sans réforme », désignant par là la manière dont une certaine forme de gouvernance contemporaine instille des changements décisifs, en privilégiant une stratégie de contournement de certains partenaires institutionnels et de valorisation de différents stakeholders (parties prenantes, acteurs non institutionnels) sans passer par le moment criti- 
que de la réforme (Vinokur, 2002), c'est donc en un autre sens qu'il convient de l'entendre concernant la période englobant la IV République ; l'explication de ce phénomène par l'instabilité ministérielle, cependant bien réelle, ne suffit pas, non plus que celle par la neutralisation réciproque dans laquelle se tenaient les deux directions ministérielles des premier et second degrés, redoublée par celle des deux organisations syndicales représentatives de ces deux secteurs concurrents (Frajerman, 2007). C'est la difficulté à trouver un accord sur une conception de l'égalité complexe (en relation avec les idéaux de l'école unique), base d'une justice elle-même complexe, source des problèmes toujours éprouvés par notre système éducatif, que nous nous proposons d'approcher centralement dans cette étude.

\section{QUATRE PROJETS DE RÉFORME : UNE DÉCLINAISON PROGRESSISTE AVANCÉE DE L'ÉGALITÉ COMPLEXE}

\section{Conditions de l'institution scolaire dans l'immédiat après-guerre}

“ La marée, en se retirant, découvre soudain, d'un bout à l'autre, le corps bouleversé de la France " (de Gaulle, 1959, p. 1). À la Libération, I'institution scolaire française doit être entièrement refondée à la suite des mesures de démantèlement prises par Vichy: suppression des écoles normales d'instituteurs et d'institutrices, atteintes portées contre l'esprit laïque (dissolution de la Ligue de l'enseignement) et mesures en faveur de l'enseignement privé, lois d'exception, enseignants frappés d'interdits professionnels et raciaux, suppression des délégués cantonaux, recrutement arbitraire des inspecteurs, suppression des organismes consultatifs, interdiction des syndicats et confiscation de leurs biens. L'attitude générale exprimée par les gouvernements de Vichy a été négative envers la démocratisation (3) ; ainsi, par la loi du 15 août 1941, le bénéfice de la gratuité a été retiré aux élèves de première, à ceux des classes terminales de philosophie et de mathématiques élémentaires, de même qu'à ceux des classes préparatoires.

Après les bombardements, on dénombre 4900 classes primaires détruites, 6000 partiellement endommagées ; 47 établissements secondaires ont été entièrement détériorés, 120 partiellement; dans l'enseignement technique, 56 collèges, ainsi que des Écoles nationales professionnelles, ont subi des dégâts plus ou moins importants ; des universités comme celle de Caen doivent être entièrement rebâties, la plupart ont été paralysées en matière de recherche et de formation (4). Aux problèmes de locaux et d'équipement, aux problèmes de recrutement s'ajoutent des problèmes pédagogiques (portant sur les contenus et les méthodes). C'est dire l'ampleur de l'effort à accomplir dans le domaine de l'éducation comme dans tous les autres. Dès le 15 mars 1944, le Conseil national de la Résistance (CNR) (5) avait énoncé son programme qui impliquait «la possibilité effective pour tous les enfants français de bénéficier de l'instruction et d'accéder à la culture la plus développée quelle que soit la situation de fortune de leurs parents, afin que les fonctions les plus hautes soient réellement accessibles à tous ceux qui auront les capacités requises pour les exercer et que soit ainsi promue une élite véritable, non de naissance mais de mérite, et constamment renouvelée par les apports populaires ". Si ce texte a bien une orientation démocratique en ce sens qu'il dénonce - comme l'a fait toute une tradition de l'école unique - la ségrégation de nature foncièrement sociale sur laquelle reposaient les études secondaires et supérieures, il s'appuie sur une certaine notion de l'égalité complexe, et ne prétend pas développer le leurre d'un accès rapide, effectif et égal de tous aux plus hautes fonctions.

\section{Une matrice réformatrice commune}

D'une certaine façon, l'état de délabrement du pays, exigeant une reconstruction matérielle et une refondation institutionnelle, aurait pu constituer une chance pour la réforme scolaire, qui n'avait pu aboutir sous le Front populaire malgré les tentatives et les quelques réelles avancées effectuées par le ministre Jean Zay, dont les réformateurs progressistes se réclament. La première ébauche de réorganisation de l'enseignement est connue sous le nom de «plan d'Alger » (6) et procède d'une commission créée le 21 janvier 1944 par René Capitant, commissaire à l'Éducation nationale et à la Jeunesse dans le Comité français de la Libération nationale, précisément implanté à Alger. Ce plan, issu d'un rapport piloté par l'universitaire Marcel Durry (7), constitue le premier projet éducatif officiel de la France Libre, et il va en quelque sorte esquisser les linéaments des textes réformateurs les plus progressistes de la période, dont le plus célèbre et le plus durable (dans les esprits, à défaut des faits) est bien sûr le plan Langevin-Wallon, rendu public en mai 1947 , et supposé suffisamment connu pour ne pas être exposé à nouveau en détail (8). Sans entrer dans le détail de chacun, on peut solidariser entre 
eux quelque quatre projets présentant des caractéristiques démocratiques communes, et revendiquant implicitement ou explicitement leur inscription dans une lignée qui comprend Condorcet, Lakanal, les Compagnons de l'Université nouvelle, le Front populaire et Jean Zay: le plan d'Alger et le plan Langevin-Wallon, ainsi que les deux projets portant le nom du socialiste Édouard Depreux, le premier élaboré au titre de ministre de l'Éducation nationale (1948) (9), le second au titre de responsable de la SFIO (1955).

Des éléments de la matrice progressiste qui tend à fédérer ces quatre textes programmatiques peuvent être ainsi répertoriés :

- accès au second degré (10) obligatoire et gratuit pour tous, prolongation de la scolarité obligatoire jusqu'à 18 ans ;

- mise en place, après la scolarité élémentaire, d'une période d'orientation, d'une durée plus ou moins longue et correspondant à un tronc commun, en vue de détecter les aptitudes, et politique d'orientation scolaire soutenue, la notion d'aptitude jouant un rôle central dans la nouvelle conception de l'articulation de l'éducation à la sphère économique et sociale ;

- à l'issue du tronc commun, orientation vers des choix différenciés (pratique, professionnel ou théorique) ;

- développement des enseignements techniques et professionnels dans le cadre d'une égale dignité des cursus et des emplois correspondants; en conséquence, démultiplication des élites (11), qui ne doivent plus être limitées aux seuls élèves des filières théoriques, et spécialement des humanités classiques ;

- suppression des examens d'entrée et intermédiaires ;

- rénovation des méthodes pédagogiques et des contenus ;

- formation professionnelle commune aux maîtres des premier et second degrés.

L'expression « égalité des chances » n'est employée à la lettre dans aucun des textes, mais l'idée en est bien présente, que nous traduirons plutôt par égalité des possibles offerts, afin d'éviter tout anachronisme. La conception sous-jacente fait désormais reposer le succès scolaire sur le seul mérite individuel, ce qui préfigure un progrès démocratique considérable par rapport à la situation antérieure, comme l'exprime ce passage de l'exposé des motifs du plan d'Alger: "Malgré des discours sonores et des bourses chiches, les cancres restaient dans les lycées et les élèves doués aux champs et à l'usine », ou cet extrait du plan Langevin-Wallon : « La sélection actuelle [...] aboutit à détourner les plus doués de professions où ils pourraient rendre d'éminents services". Dans la dissociation entre réussite ou échec scolaire et type d'appartenance sociale, qui peut nous surprendre (voire nous indigner) rétrospectivement, il faut sans doute moins voir une naïveté sociologique, très explicable, que l'ampleur du déficit démocratique, devenue inimaginable à nos yeux, en matière d'accès aux études secondaires des élèves des catégories populaires - malgré quelques progrès en ce sens que révèle la mixité sociale de certains collèges pendant l'entredeux-guerres (Prost, 2006) -, en même temps que le rayonnement dans la société du succès de quelques élèves boursiers. Remédier à ce déficit par la simple ouverture à tous de l'accès au second degré pouvait donner l'illusion d'une démocratisation automatique, dont le plan Langevin-Wallon apparaît emblématique, encore qu'il manifeste bien la conscience des difficultés à réaliser immédiatement ses ambitions.

La volonté démocratique de former les nouvelles et multiples élites en puisant dans toutes les classes de la société grâce à un enseignement de masse et de haut niveau s'accompagne de la confiance en une possibilité d'accord spontané entre les besoins sociaux et la répartition des aptitudes individuelles. Cette adéquation rêvée entre les aptitudes et la structure de l'emploi par la médiation de l'éducationformation risque là encore de nous apparaître largement illusoire aujourd'hui, mais l'idée peut être mieux comprise si on la replace dans le contexte socio-économique de l'époque qui est celui de la reconstruction et des besoins en matière de travail qualifié exigés par la modernisation du pays. II n'en reste pas moins que le texte postule une sorte d'harmonie préétablie, dont la démocratisation libérerait enfin réellement les potentialités : "L'introduction de la justice à l'école mettra chacun à la place que lui assignent ses aptitudes pour le plus grand bien de tous. La diversification des fonctions sera commandée non plus par la fortune ou la classe sociale mais par la capacité à remplir la fonction. La démocratisation de l'enseignement, conforme à la justice, assure une meilleure distribution des tâches sociales ».

\section{La notion d'aptitude, pièce centrale d'un dispositif d'égalité complexe}

En référence explicite aux orientations éducatives du CNR, la notion d'aptitude combinée au «principe de justice » joue un rôle central dans le projet 
Langevin-Wallon considéré spécifiquement : « Le premier principe, celui qui par sa valeur propre et l'ampleur de ses conséquences domine tous les autres est le principe de justice [...] Tous les enfants, quelles que soient leurs origines familiales, sociales, ethniques ont un droit égal au développement maximum que leur personnalité comporte. Ils ne doivent trouver d'autre limitation que celle de leurs aptitudes ». C'est en raison de ce caractère central que nous avons estimé pertinent d'interroger d'un peu plus près le fonctionnement de la notion d'aptitude dans le texte du projet, en recourant notamment à une analyse lexicométrique (12).

Sur les 15507 occurrences constituant le texte du projet issu de la commission (sans le texte de l'appendice), on ne s'étonnera pas que les trois mots les plus employés soit par ordre décroissant: « enseignement(s) », « enfant(s) », « maître(s) ». « Aptitudes » offre quarante-deux occurrences, presque à égalité avec «éducation", juste derrière «formation », et devant « études ». C'est dire son importance fonctionnelle. Le texte ne propose aucune définition du concept à caractère scientifique, mais une rapide analyse lexicale fait apparaître une acception dynamique et évolutive de la notion, fondée sur l'idée d'une éducabilité essentielle et permanente de l'humain. Le terme « dons » n'apparaît qu'une fois, et l'adjectif «doués » deux, ce qui est révélateur du refus de toute orientation psychologique fixiste. Pour autant, malgré cette rupture avec la notion de dons et malgré l'insistance sur l'éducabilité permanente, soulignée par l'importance accordée à l'éducation post-scolaire, nous ne pouvons manquer aujourd'hui d'interroger la distinction non remise en cause, parce que déterminant - à partir de l'âge de quinze ans - les options terminales de la scolarité obligatoire, entre aptitudes manuelles et aptitudes intellectuelles, dont quarante ans de sociologie critique nous ont montré le caractère socialement construit. Certes, on doit aussi relever que le projet raisonne en termes de processus évolutifs, et se place dans une perspective de transformation des fondements de la division sociale du travail, supposant une redistribution possible et souhaitable de la hiérarchie admise entre tâches intellectuelles et manuelles.

Avant toute intervention, dans la décennie suivante, précisément de cette sociologie critique qui modifiera le regard sur la nature de «la justice » scolaire ainsi produite, on saisit les composants idéologiques fondateurs de la matrice des projets de réforme progressiste les plus avancés. Ceux-ci ont nécessairement à voir avec la notion d'une égalité complexe, poussant le curseur le plus loin qu'il est alors envisageable en direction de l'égalité simple (conçue comme idéal régulateur) : élargissement à tous les enfants de l'accès à un enseignement secondaire prolongé, sans distinction de catégories sociales ; recherche de l'égalité maximale des possibles offerts, en s'appuyant sur la détection et le développement optimal des aptitudes au cours d'une scolarité obligatoire longue; mais égalité vouée à susciter dans son mouvement même de l'inégalité, puisque explicitement articulée, sans remise en cause de l'évaluation individuelle, à la réhabilitation de la méritocratie (différenciation désormais considérée comme juste car en principe fondée - du fait de la redistribution de la donne sociale à l'école - sur le seul critère du mérite individuel, pur en quelque sorte). On pourrait ici risquer l'expression rawlsienne d'inégalités justes (Rawls, 1987), les plus justes qu'il soit possible d'envisager sous un certain angle, situées par les rapporteurs dans la perspective de transformations sociales démocratiques de grande ampleur. Quant à eux, les partisans d'une éducation égalitariste dite intégrale (Mole, 2007) n'y trouveraient pas leur compte.

\section{ENTRE L'ÉGALITÉ COMPLEXE ET SES MARGES, ET UNE SORTIE RÉSOLUE DU PROJET D'ÉGALITÉ}

Nous examinons maintenant un groupe de cinq projets occupant sur le curseur de la démocratisation des positions s'éloignant plus ou moins des précédentes, et plus spécialement de celles du plan Langevin-Wallon, quoique plusieurs se réclament de lui : les projets d'Yvon Delbos (1949), de la direction du second degré du ministère de l'Éducation nationale (1953), de la direction du premier degré ministère (1953), d'André Marie (1953), de René Billères (1956). Sitôt après sa publication et sa non mise en œuvre, le plan de 1947 a en effet acquis un prestige " presque sacral », devenant " la référence majeure et quasi liturgique de la gauche en matière de réforme de l'enseignement " selon les expressions d'Antoine Prost (1968, p. 420).

Deux projets d'origine ministérielle donnent l'impression de vouloir prolonger la scolarité obligatoire jusqu'à dix-huit ans (Delbos, Marie) mais il s'agit d'un leurre puisque, dans les deux cas, après un cycle d'orientation et d'initiation de deux ans, une bifurcation définitive est prévue à treize-quatorze ans pour certains élèves «qui ne se destinent pas à l'ensei- 
gnement du second degré »(Delbos, art. 12). Selon l'exposé des motifs assez ambigu du projet Delbos, ces élèves sont appelés à poursuivre une éducation déjà qualifiée de post-scolaire, "simultanément ou successivement " dans les cours complémentaires existants, et dans les cours post-scolaires publics qui doivent aussi accueillir des travailleurs adultes. Au regard des critères évoqués plus haut, on peut avancer que le pacte d'une égalité complexe est en ce point rompu, certains enfants étant écartés très tôt de l'espace commun, à l'aide d'un argument qui deviendra un classique : "vouloir obliger tous les enfants à entreprendre et à poursuivre des études qui exigent des dons que la nature [...] est loin d'impartir également à chacun, c'est courir le risque [...] d'abaisser le niveau de l'enseignement... ". Partisan d'un élargissement des bases sociales du recrutement («II faut jeter les filets de l'instruction sur le peuple entier pour pêcher toutes les intelligences " a-t-il déclaré au congrès de son parti à Nice en 1925), le ministre radical, normalien supérieur et agrégé, qui a été un des sept titulaires éphémères de l'Instruction publique sous le Cartel des gauches, a néanmoins une conception restrictive de l'égalité (13) et reste solidaire d'une vision élitiste du second degré. En cela il s'inscrit dans un courant idéologique tentant d'allier certaine ouverture démocratique et tradition française du secondaire, que nous proposons de dénommer le démo-élitisme. Cette conception se différencie de ce qu'on appelle communément l'élitisme républicain : tandis que ce dernier vise plutôt à dégager une élite de l'école du peuple au sein d'un continuum premiersecond degré incluant la possibilité d'une adaptation des programmes et des méthodes pédagogiques du secondaire aux nouveaux élèves qui le fréquentent, le démo-élitisme - tout en acceptant l'élargissement des bases sociales du recrutement des élèves - pose d'emblée et a priori l'autonomie, la supériorité et la distinction du vrai secondaire (14), qui ne doit pas fondamentalement changer ses méthodes, comme l'a exprimé avec force le directeur de l'enseignement secondaire Jean Delvolvé dès la fin des années 1920 (Delvolvé, 1928). D'ailleurs, dans le projet de 1949, le second degré ne commence vraiment qu'au terme du cycle d'orientation.

Dans le texte signé André Marie (1897-1974) (15), les choses se présentent de manière encore plus ambiguë. À l'issue d'un processus un peu compliqué en plusieurs étapes, ce texte ministériel résulte des propositions de réforme présentées par le directeur général du second degré Charles Brunold, modifiées par le Conseil supérieur du 24 juin 1953. Ce projet de décret relatif à l'enseignement du second degré mérite d'être considéré un instant ; Charles Brunold, qui succède à Gustave Monod (Chapoulie, 2005), n'a manifestement pas les mêmes références progressistes que ce dernier. II se propose d'instituer, après le cycle d'orientation en $6^{\mathrm{e}}$ et $5^{\mathrm{e}}$, deux cursus secondaires distincts, l'un court (second degré spécial) et l'autre long (second degré normal). Une telle mesure de séparation, survenant très tôt dans la scolarité secondaire, se situe au centre d'enjeux de la plus grande importance et aura une postérité dans les premières réformes de la $V^{e}$ République. En effet, pour Charles Brunold, qui souligne la disparité entre le nombre d'élèves du secondaire public («550 $000 »)$ et celui d'élèves des centres d'apprentissage («150 000 ") et invoque les "vrais besoins de la nation ", l'essentiel semble résider dans le constat selon lequel «nos facultés sont encombrées d'étudiants qui ne sont pas tous parfaitement capables de recevoir l'enseignement qui leur est offert », situation qu'il s'agit d'éviter à l'avenir, en l'anticipant dès le début du secondaire. La voie choisie est ici délibérément celle de l'élitisme, qui, ne montrant aucune conscience de la corrélation entre études longues et origine sociale, ne prend même pas soin de recommander un élargissement des bases sociales du recrutement. Pour résoudre les problèmes posés par "l'explosion scolaire", résultant de l'accroissement démographique, de la demande sociale d'éducation et des besoins objectifs de la société (Cros, 1961), il faudrait organiser deux réseaux parallèles en développant la fréquentation du cycle court et de l'enseignement technique, et en réservant le cycle long à une minorité. On a ici rebasculé, hors toute problématique de l'égalité complexe, dans la perpétuation des inégalités scolaires, non sans avoir cependant conçu une courte période de tronc commun (un an).

Il convient de relever que, sitôt rendu public ce projet de la direction du second degré, celle du premier degré s'est sentie obligée de répliquer avec le sien propre, tant est exacerbée la rivalité entre les deux secteurs et tant la concurrence est rude pour savoir qui des professeurs ou des instituteurs a vocation à enseigner dans les classes de $6^{\mathrm{e}}$ et de $5^{\mathrm{e}}$. Cette tension primaires/secondaires se voit d'ailleurs redoublée par une opposition syndicale paradoxale, les deux principales organisations - le Syndicat national des instituteurs (SNI) et le Syndicat national de l'enseignement secondaire (SNES) - appartenant alors à la même fédération, la Fédération de l'Éducation nationale, et à la même tendance idéologique en son sein (Frajerman, 2007 ; Robert, 2004). Le projet de la direction du premier degré met notamment l'accent sur la nécessité de multiplier dans les 
campagnes les cours complémentaires d'orientation à deux classes rattachés à une école primaire, ainsi que, ailleurs, les cours complémentaires à cinq classes, qui conduisent jusqu'au terme de la classe de seconde, parcours sanctionné par un diplôme particulier. Dans ces établissements enseignent en effet exclusivement des personnels du premier degré.

On comprend que, dans ces conditions, la synthèse incombant au ministre n'ait pas été facile à réaliser. Si la scolarité jusqu'à dix-huit ans est bien évoquée dans l'article 1 du projet de loi Marie, ce n'est pas sur le mode de l'obligation, et en réalité l'enseignement ne reste obligatoire que jusqu'à quatorze ans. L'orientation est organisée en $6^{\mathrm{e}}$ et $5^{\mathrm{e}}$, classes qui appartiennent au second degré, mais «qui donnent dans les divers établissements un enseignement conforme à la vocation de ces établissements " (article 11). Autrement dit, même si formellement le second degré s'applique à tous les enfants fréquentant tous types d'établissements (parmi lesquels les cours complémentaires annexés aux écoles primaires), le texte admet explicitement que la nature de l'établissement où est dispensé l'enseignement doit déterminer la nature (au fond primaire ou secondaire) de cet enseignement, ce qui revient à entériner un ordre immuable des choses. C'est un dispositif que l'on retrouvera dans le décret Berthoin du 6 janvier 1959 mais le tropisme établissement sera alors relativement masqué, dans un contexte très volontariste, au profit d'une espérance d'homogénéisation. Malgré quelques mesures d'orientation plus progressiste, le projet de loi Marie, indifférent à un recrutement élargi pour un second degré de pleine valeur, ne semble ainsi nullement pouvoir prendre place dans le segment délimitant une problématique de l'égalité complexe.

Même s'il en rabat par rapport aux exigences posées par le Plan Langevin-Wallon, désormais référence incontournable, le projet gouvernemental de loi-cadre (16) signé par René Billères s'inscrit indéniablement, quant à lui, sur ce segment. Revendiquant sa filiation avec l'école unique, et au-delà avec les idéaux de la Révolution française, il constate à son tour, douze ans après le plan d'Alger: "Notre système scolaire, qui repose trop étroitement encore sur le principe de la sélection d'une élite peu nombreuse choisie pour la plupart en dehors des couches populaires, ne répond pas au vœu unanime des Constituants de 1946... ». L'exposé des motifs est le premier à être bardé de notations à caractère sociologique (mention des travaux de l'Institut national d'études démographiques ; critique, soutenue par des statistiques, des critères socio-économiques déter- minant les destinées scolaires) ; il fait également allusion aux réformes étrangères alors en cours (Suède, USA, URSS).

Si l'expression «égalité des chances » n'apparaît pas à la lettre, le projet entend « placer pratiquement tous les enfants, quelle que soit leur origine sociale, dans les conditions d'égalité pour l'accès à toutes les formes de culture ». Pour cela, il prévoit une scolarité obligatoire jusqu'à seize ans, et un tronc commun de deux ans, total pendant la première année, partiel ensuite (enseignement commun de certaines matières seulement). C'est le terme d' "école moyenne» qui sert à définir ce moment de la scolarité consacré à l'orientation, évitant la focalisation sur la question de son appartenance ou non au second degré, et solution déclarée minimum pour permettre à la scolarité de type lycée une durée suffisante. À l'issue du tronc commun (à treize ans), sont en effet prévus des enseignements diversifiés correspondant à l'école dite «terminale », au collège ou au lycée, ces filières d'inégale valeur étant cependant réunies, autant que possible, dans des établissements communs. Tout en étant moins ambitieux que le plan Langevin-Wallon et ses succédanés directs, le projet socialiste de $R$. Billères constitue ainsi une nouvelle variante dans la voie de l'égalité complexe, sans doute plus proche d'un réalisme dicté par les contraintes politiques et économiques du moment.

Pour autant, quoique approuvé par le Conseil supérieur, et préparé par certaines mesures prises dans le même esprit, pas plus que les autres ce projet n'a abouti ; les raisons de son échec peuvent apparaître exemplaires pour toute la période considérée. Si les instituteurs et leur syndicat sont globalement favorables à cette réforme, surtout à cause de l'institution d'une école moyenne dont ils espèrent avoir le monopole, les professeurs se montrent beaucoup plus réservés et les plus conservateurs d'entre eux franchement hostiles, le SNES n'hésitant pas à s'allier avec ces derniers. Ils bénéficient du relais, à l'Assemblée, des députés agrégés, l'école moyenne et le tronc commun étant dénoncés comme une mutilation de l'enseignement secondaire (Dreyfus, 1965). De même, les parents d'élèves des lycées et collèges s'inquiètent d'un tronc commun qui n'est pas un vrai secondaire. L'enseignement catholique redoute la concurrence que lui ferait une prolongation de la scolarité obligatoire gratuite, s'il devait ne pas recevoir les aides financières permettant à la liberté qu'il prétend incarner de s'exercer réellement... À la variété des déclinaisons possibles de l'égalité complexe (qu'est-ce qui est juste, ou le moins injuste possible, 
au moment « $t$ »?) s'ajoute une variété presque indéfinie des critiques, fonction des intérêts particuliers des catégories d'acteurs. Les exécutifs faibles de la IVe République n'étaient pas en mesure de dominer cette dispersion, qui s'est manifestée dans des configurations variables à l'occasion de chaque projet.

\section{LES TEXTES BERTHOIN MIS EN PERSPECTIVE}

C'est, comme on le sait, le ministre radical Jean Berthoin (1895-1979) qui réussira enfin à mettre en place une réforme scolaire, le 6 janvier 1959, dans ce qui est en fait le dernier gouvernement de la IVe République dirigé par le général de Gaulle. Mais ces décret et ordonnance ont fait date au titre des premières décisions éducatives de la $V^{e}$ République, dont Charles de Gaulle devient officiellement président le 8 janvier.

Jean Berthoin avait déjà été ministre de l'Éducation nationale sous deux gouvernements successifs de la IV République, les gouvernements Pierre Mendès-France et Edgar Faure (17). C'est au nom de ce dernier qu'il a présenté un projet de loi portant réforme de l'enseignement public en août 1955, en s'appuyant sur les travaux préalables d'un comité d'étude dirigé par le recteur Jean Sarrailh entouré entre autres de plusieurs industriels. Les deux textes présentent des similitudes, notamment dans leur référence commune à des priorités d'ordre économique, avant les priorités tenant à la justice sociale, même si celles-ci ne sont pas ignorées. Ce n'est en effet pas un hasard si J. Berthoin, sénateur, a établi en 1952 un rapport général sur la question économique auprès du Conseil de la République (18), rapport repris dans la politique financière du parti radical et radical-socialiste en 1953.

À l'inverse de certains argumentaires, J. Berthoin déclare dans l'exposé des motifs de 1955: «Nous n'avons pas trop d'étudiants... Notre faiblesse et notre embarras proviennent, non de leur nombre excessif, mais de leur mauvaise répartition » $(58 \%$ d'étudiants en lettres, seulement $26 \%$ en sciences). Or les besoins du pays concernent désormais largement le secteur tertiaire (professions libérales, enseignants, chercheurs, carrières commerciales et scientifiques) tout en exigeant un accroissement de qualification dans le secteur secondaire (agents techniques, techniciens, techniciens supérieurs). Face à tous ces emplois insuffisamment pourvus, il y a nécessité d'un interventionnisme de l'État (qui sera encore plus marqué en 1959 dans le contexte gaulliste) pour ne plus laisser au hasard l'orientation des élèves, dans le cadre de l'accentuation de l'explosion scolaire. Toutes les mesures envisagées (1955) puis réellement décidées (1959) vont découler de cette analyse économique.

Nous rappelons les principales dispositions arrêtées (1959), sans nous attarder sur les différences entre les deux textes, les principales étant, en 1955, la volonté de placer un examen d'entrée dans l'enseignement long au terme des deux années d'orientation, et la suppression du baccalauréat :

- prolongation de la scolarité obligatoire jusqu'à seize ans, devant prendre effet en 1967 ;

- institution d'un cycle d'observation d'une durée de deux ans, pour tous, couvrant les classes de sixième et de cinquième, avec un tronc commun sans latin limité au premier trimestre de sixième ;

- institution, auprès des classes ou groupes de classes, d'un conseil d'orientation composé des enseignants dont la tâche sera de "préparer, grâce à l'observation très suivie dont les élèves auront été l'objet, les choix qui interviendront à la fin de la deuxième année du cycle, entre les différents types d'enseignement »;

- à l'issue du cycle d'observation, possibilité de répartition des élèves entre cinq types d'enseignement en fonction de leurs « aptitudes » (enseignement général long, classique ou moderne ; enseignement technique long; enseignement général court; enseignement technique court; enseignement terminal conduit avec les professions).

Il est décidé d'installer le cycle d'orientation commun à tous les élèves dans des établissements demeurant différents: premiers cycles des lycées et collèges ; ex-cours complémentaires, désormais appelés collèges d'enseignement général, collèges d'enseignement technique et écoles primaires. Du fait du maintien de ce cloisonnement entre les types d'établissements et des risques évidents de ségrégation qu'il contient, le réformateur prévoit le principe de " classes passerelles » destinées à offrir la possibilité d'une circulation d'élèves d'un secteur d'enseignement à un autre.

L'objectif déclaré de ces mesures réformatrices vise d'abord à « assurer une prospection aussi complète que possible de nos ressources juvéniles », c'est-àdire à élargir très considérablement le vivier social des jeunes susceptibles de recevoir une formation secondaire. Autrement dit, s'opère dans cette réforme 
la rencontre d'une nécessité économique avec un accroissement de la justice sociale, mais si les deux coïncident, la motivation déclarée la première n'est pas celle de l'égalité sociale.

Les mots-clés de l'exposé des motifs de 1959 sont en effet sans ambiguïté : investissement éducatif, ressources humaines, aptitudes, réserves d'aptitudes; on trouve même en titre de paragraphe: investir à plein profit. Ces termes ressortissent tous au vocabulaire de la théorie du capital humain, qui connaît alors une diffusion internationale (Robert 2006a).

En même temps s'affirment, d'une manière très insistante, la volonté de ne plus laisser à l'arbitraire individuel le choix des filières à suivre, en redéfinissant les critères de l'excellence du côté des mathématiques, des sciences et des techniques, d'une part, et la nécessité de pratiquer une sélection impitoyable, d'autre part : "Nous ne pouvons plus maintenir une organisation scolaire qui ne nous permet de former qu'un chercheur, un ingénieur, un professeur quand il en faudrait deux, un technicien quand trois seraient nécessaires tandis qu'à l'inverse, se presse dans nos enseignements supérieurs des lettres, de la philosophie et du droit une foule d'étudiants à qui nous n'avions pas préparé d'autre issue, et qui doivent maintenant recourir à de tardives et difficiles reconversions » (§ 3$)$. En cela, les dispositions de la réforme s'accordent parfaitement avec les recommandations du III Plan (1958-1961) qui, outre l'augmentation nécessaire du nombre des ouvriers spécialisés et des ouvriers qualifiés, met l'accent sur les besoins en ingénieurs, techniciens et cadres, dans la ligne même de la conception économique évoquée plus haut.

On dénombre trente et une mentions de termes ou syntagmes appartenant à la série paradigmatique "aptitude » dans les quinze colonnes qu'occupe au Journal officiel de la République française du 7 janvier 1959 l'exposé des motifs valant pour l'ordonnance et le décret. De même, au seul titre II intitulé «Du cycle d'observation », sur vingt et un courts articles et soixante-dix lignes au total, "aptitude » revient cinq fois. La psychologie à prétention différentielle entre ainsi en relation avec la volonté d'élargir les bases sociales du recrutement secondaire et, tout à la fois, celle de sélectionner sévèrement les élèves en fonction des capacités de chaque filière, elles-mêmes déterminées par les besoins économiques et les perspectives d'emplois. Le souci de ne pas laisser détourner la réforme de tels objectifs est omniprésent: "Comment accepter la perspective de lycées bientôt submergés par un million d'élèves, dont la moitié sans doute n'y seraient entrés qu'en méconnaissant leurs véritables aptitudes ? Le drame est là : nous retenons dans l'enseignement théorique nombre de jeunes esprits qui trouveraient mieux leur voie dans l'enseignement technique »

Si l'esprit de 1959 apparaît moins libéral que celui de 1955, c'est que C. de Gaulle et ses conseillers sont passés par là, qui théorisent plus fortement l'idée de sélection, pour peu qu'elle repose sur des bases de justice minimale. On peut aussi constater que les mesures arrêtées essaient de composer, autant que faire se peut, avec certains aspects des tentatives antérieures de conception du tronc commun (en traitant cependant la déclinaison présente de celui-ci a minima, puisque le réduisant en fait à un trimestre) et avec certaines des critiques prévisibles, en donnant cependant des gages aux conservateurs de l'ordre secondaire.

Peut-on alors dire que les textes Berthoin s'inscrivent sur le segment de l'égalité complexe ou qu'ils restent en deçà ? Nous prenons ici appui sur une comparaison avec les grandes lignes du Plan Langevin-Wallon. Le fait que des décisions ont été effectivement prises et vont engager la France, de manière chaotique mais réelle, sur la voie d'une démocratisation au moins quantitative, inclinerait à répondre positivement. Une analyse rapide du rapport opéré, dans les deux documents, entre aptitudes individuelles et répartition des personnes dans la division du travail pourrait plaider dans le même sens. Une confrontation plus approfondie oriente néanmoins vers une autre réponse.

La perspective dans laquelle le projet de 1947 situe le concept d'aptitude (19) a manifestement un caractère dynamique et évolutif, là où le décret de 1959 allie fixisme et naturalisation. D'après Langevin-Wallon : "L'étude objective de chaque individualité [...] se fera dans le respect de la personnalité enfantine, afin de développer en chacun les aptitudes originales [...] Le but de l'enseignement est premièrement d'assurer aux aptitudes tout le développement dont elles sont susceptibles ». Selon Berthoin : “L'orientation [est] fondée sur la pleine observation [des] aptitudes [...] L'enseignement [est] le cadre et le moyen de l'observation et de la détection des aptitudes "; il n'est pas question ici de développement d'aptitudes en germe, mais de détection de celles qui sont déjà formées, déjà données par la nature. De fait, la durée de la scolarité obligatoire jusqu'à dix-huit ans laisserait objectivement plus de temps pour développer les qualités propres à chacun qu'une obligation jusqu'à seulement seize ans, exigeant d'aller vite en matière d'orientation. Certes, la mesure effectivement 
prise par le pouvoir gaulliste a constitué un incontestable progrès qu'aucun gouvernement précédent n'avait réalisé mais, d'un autre côté, des dérogations à la règle commune relativisaient immédiatement ce progrès en organisant des sorties précoces : « La formation professionnelle qui peut achever la scolarité obligatoire " peut être donnée, hors de tout lien avec l'école, « dans des entreprises » (art. 31 du décret du 6 janvier 1959).

En 1960, la critique qu'Henri Wallon a lui-même été amené à faire de la réforme scolaire porte justement sur cette organisation d'une différenciation hâtive des élèves par types d'enseignement, c'est-à-dire de la sélection - bien caractéristique de l'intention gaullienne, sur fond d'égalité formelle - que le décret s'empresse de mettre en œuvre dès la fin du premier trimestre en sixième, et dès la fin de la cinquième : "Que l'effectif de la future élite se rétrécisse avec les années de scolarité n'est pas pris comme un signal d'alarme, comme la preuve d'un échec pédagogique, mais comme un fait fatal et naturel [...] La solution est cherchée dans des enseignements courts, dans des enseignements réduits aux rudiments indispensables » (20).

\section{LA QUATRIÈME RÉPUBLIQUE ET LE SECOND DEGRÉ : DES CHANGEMENTS AFFECTANT L'ÉGALITÉ ET LA JUSTICE ?}

Depuis la fin du XIX ${ }^{e}$ siècle, la conception de ce qui est juste en matière scolaire n'a pas toujours été liée avec la recherche d'une forme d'égalité. La séquence historique où des acteurs multiples s'emploient à installer dans le second degré (du moins dans sa première phase) la problématique de changements en vue d'une égalité complexe, peut être étendue, en amont de la période 1944-1959, au gouvernement du Front populaire (Chapoulie, à paraître). Néanmoins, il semble bien que le vrai saut quantitatif et qualitatif se soit produit, pour de multiples raisons, après la seconde guerre mondiale.

Le taux de scolarisation de la population de douze à quinze ans est ainsi passé de $20,5 \%$ à la Libération à $44 \%$ en 1957-1958, gagnant cinq points entre 1945 et 1950 et quinze points entre 1950 et 1956 . À partir de 1951-1952, le nombre des élèves inscrits en sixième s'est considérablement accru alors même qu'il s'agissait, jusqu'en 1956-1957, d'enfants nés pendant la guerre, à un moment où le taux de natalité était bas. Ce phénomène révèle donc un effet de sco- larisation, avant tout effet simplement démographique. II faut d'autre part relever que la préscolarisation en maternelle a augmenté de manière très importante et que, dans l'enseignement supérieur, les effectifs sont passés de 123300 étudiants en 1945-1946 à 240000 en 1960. On assiste également à l'accroissement général de la scolarisation dans le technique et professionnel, public et privé - de 360000 élèves en 1944 à 500000 dès 1951 (Charlot \& Figeat, 1985, p. 357). Sans être exhaustif, ce bref recensement des augmentations d'effectifs au cours de la décennie 1950-1960 donne la mesure des changements quantitatifs intervenus. Les gouvernements, malgré l'échec de tous leurs projets réformateurs d'envergure, ont dû non seulement absorber ces évolutions, mais bien pour une part les anticiper et les stimuler. La prolongation de la scolarité au delà de quatorze ans, ayant ainsi commencé à s'inscrire dans les faits avant d'être reconnue en droit (1959), il a fallu des établissements nouveaux ou rénovés pour accueillir les jeunes et des enseignants pour les encadrer, donc une politique et un budget. Cela s'est traduit entre autres par l'augmentation des crédits consacrés aux constructions et aux équipements scolaires, une commission spécialisée ayant été installée auprès du Commissariat au Plan à partir de 1951 (Commission Le Gorgeu). Le II Plan (1954-1958) a intégré un plan d'équipement scolaire, universitaire, scientifique, artistique et sportif. L'évolution comparée du budget de l'Éducation nationale en pourcentage du budget général de l'État montre une progression de 7,4 \% en 1952 à 10,2 \% en 1958. Cet effort budgétaire place la France parmi les pays occidentaux dont le taux de progression des dépenses d'éducation est alors le plus fort.

Jouant leur rôle dans la recherche de plus de justice, des changements qualitatifs significatifs eurent parallèlement lieu: expérimentation d'innovations sous la forme des "classes nouvelles", faisant suite aux «classes d'orientation » voulues par Jean Zay (Robert, 2006b) ; unification des programmes et des examens au niveau du premier cycle du second degré entre sections des cours complémentaires, des lycées et collèges, et sections générales des collèges techniques; suppression de l'examen d'entrée en sixième ; développement de l'enseignement professionnel avec la création des centres d'apprentissage et des Écoles normales nationales d'apprentissage (ENNA) - chargées de la formation des professeurs de l'enseignement professionnel-, développement de la formation des maîtres : création des conseillers pédagogiques, du Certificat d'aptitude du professorat de second degré (CAPES) et des Centres pédagogiques régionaux pour la formation des profes- 
seurs du second degré (CPR) ; création d'un véritable corps de psychologues scolaires, etc. Bref, le bilan de la période n'est pas négligeable. S'il s'est montré impuissant à faire la ou les réformes éducatives importantes, l'État a accompagné, et même encouragé, la demande sociale portant sur plus d'éducation, plus d'ouverture de l'accès au second degré, et donc plus d'égalité.

Si l'on ajoute à ces mesures réelles, quoique fragmentaires, l'intensité de la volonté réformatrice et de la réflexion qu'elle a suscitée, il est clair qu'au terme de la période la question de l'égalité complexe et de la justice scolaire ne se présente plus comme avant ; celle-ci est devenue une urgence de l'agenda politique, et la résolution du problème semble désormais se présenter autant comme une exigence découlant du tournant accompli par la société et l'économie que comme une exigence morale. La manière de poser le problème autorise beaucoup moins qu'auparavant les argumentaires procédant d'autres conceptions républicaines de la justice, non fondées sur l'égalité (Dubois, 2007), que les Compagnons et les partisans de l'école unique avaient commencé d'attaquer dès la fin de la précédente guerre (Garnier, 2007). Entre 1944 et 1959, on est passé, à travers plusieurs varian- tes, d'un point de vue à un autre, les deux se réclamant d'une mise en relation de la notion de justice avec celle d'égalité, implicitement, et quasi nécessairement, définie comme complexe. Dans l'un, est postulée une rencontre harmonieuse entre le développement maximal des aptitudes individuelles et les besoins de l'économie (priorité restant à la culture "de l'homme, du travailleur, du citoyen»). Dans l'autre point de vue, ce sont les besoins économiques qui commandent la recherche certes la plus large possible des aptitudes, mais combinée à une sélection rigoureuse et précoce (priorité revenant alors à l'économie). S'il est possible de souligner une compatibilité de ces deux orientations avec une déclinaison plus ou moins ambitieuse des idées de démocratisation et d'égalité complexe, il demeure entre elles une indéniable différence qualitative, l'une pouvant se prévaloir de plus de justice conçue en termes idéaux, l'autre étant plus en mesure d'arguer de son efficacité pragmatique.

André D. Robert

andre.robert@univ-lyon2.fr Université Lumière-Lyon 2

UMR Éducation \& politiques (INRP-université Lumière-Lyon 2)

\section{NOTES}

(1) Rapport général sur les travaux de la commission pour la réforme de l'enseignement présenté par Marcel Durry. Alger : 21 janvier 1944 [I. motifs et tendances].

(2) Nous retenons : 1944, plan d'Alger ; 1947, plan Langevin-Wallon ; 1948, projet Depreux ; 1949, projet Delbos ; 1951-1953, projet de la direction du second degré ; contre-projet de la direction du premier degré ; 1953, projet Marie ; 1955, projet socialiste Depreux 1955 , projet Berthoin ; 1956, projet Billères. Pour la reproduction et la présentation de tous ces textes, l'ouvrage indispensable reste celui de L. Decaunes \& M.-L. Cavalier (1962).

(3) Autre chose est l'effet démocratisant involontaire entraîné par certaines décisions, comme l'intégration des Écoles primaires supérieures dans le secondaire au titre de collèges modernes (1941), ainsi que l'a montré A. Prost (2004, p. 255-261).

(4) L'Éducation nationale, n² 23 du 30 septembre 1954.

(5) Fondé le 27 mai 1943 par Jean Moulin, délégué du général de Gaulle.

(6) Publié au BOEN $n^{\circ}$ spécial 118-555 du 16 novembre 1944. Reproduit dans M. Allaire \& M.-T. Frank (1995).

(7) Maître de conférences à l'université de Paris, auteur d'ouvrages tels que Éloge funèbre d'une matrone romaine et Pline le jeune.

(8) Pour le texte original lui-même, voir G. Mialaret (1997).

(9) E. Depreux a été ministre de l'Éducation nationale du 22 janvier 1947 jusqu'au 26 juillet 1948, sous les gouvernements Ramadier puis Schuman.

(10) Pour plus de clarté nous parlons ici de second degré, en référence à la tradition, alors même que le plan Langevin-Wallon parle de $2^{\mathrm{e}}$ et $3^{\mathrm{e}}$ cycles du premier degré.

(11) «L'équité exige la reconnaissance de l'égale dignité de toutes les tâches sociales, de la haute valeur matérielle et morale des activités manuelles, de l'intelligence pratique, de la valeur technique. Ce reclassement des valeurs réelles est indispensable dans une société démocratique moderne dont le progrès et la vie même sont subordonnés à l'exacte utilisation des compétences » (Plan Langevin-Wallon).

(12) Logiciel Lexico 1, mis au point par André Salem.

(13) « Être démocrate [...] c'est organiser, dans la justice, les nécessaires hiérarchies, en fonction des aspirations humaines, des besoins nationaux et des aptitudes individuelles " déclare-t-il dans le journal La Dordogne républicaine du 14 octobre 1925. Cité dans B. Lachaise (1993).

(14) Ce n'est par pour rien que le laboratoire du démo-élitisme nous semble avoir été constitué par le micro-milieu des professeurs des classes élémentaires des lycées. Cf. A. Robert (2005).

(15) Radical-socialiste, ministre de l'Éducation nationale de septembre 1951 à juin 1954, il a attaché son nom à une loi permettant aux élèves du privé d'avoir des bourses d'État.

(16) Gouvernement Guy Mollet à majorité socialiste.

(17) Gouvernement Mendès France, du 19 juin 1954 au 22 janvier 1955 ; Faure, du 23 janvier 1955 au 31 janvier 1956.

(18) Assemblée Nationale, $2^{\mathrm{e}}$ législature, session de 1955, annexe au procès-verbal de la séance du 4 août 1955.

(19) Henri Wallon : « II n'y a pas d'aptitude dont la source soit purement endogène et, par conséquent, isolable de son objet, détachable de son milieu...Croire que, chez l'enfant, les aptitudes se développent spontanément est non seulement erroné, mais encore prive l'éducateur de toute possibilité d'action " (1952).

(20) Préface d'H.Wallon à la réédition du projet de réforme de 1947 par l'UFU. 


\section{BIBLIOGRAPHIE}

Allaire M. \& Frank M.-T. [éd.] (1995). Les politiques de l'éducation en France. Paris : La Documentation française.

Barreau J.-M. \& Legrand L. (1998). L'école unique de 1914 à nos jours. Paris : PUF.

Chapoulie J.-M. (2005). «Entre le lycée d'élite et le lycée de masse : Paul Langevin, Gustave Monod et les réformes de l'enseignement secondaire de 1936-1939 et de 1944-1951 ». In P. Caspard, J.-N. Luc \& P. Savoie (dir.), Lycées, lycéens, lycéennes: deux siècles d'histoire. Lyon: INRP, p. 145-157.

CHAPOULIE J.-M. (à paraître). "Réorganisations institutionnelles et croissance de la scolarisation post-obligatoire, 1936-1962 ». Revue d'Histoire moderne et contemporaine.

Charlot B. \& Figeat M. (1985). Histoire des ouvriers. Paris : Minerve.

Cros L. (1961). L'explosion scolaire. Paris : CUIP.

Decaunes L. \& CaValieR M.-L. (1962). Réformes et projets de réformes de l'enseignement français de la révolution à nos jours (1789-1960) : étude historique, analytique et critique. Paris: Publications de l'Institut pédagogique national.

DeLvolvÉ J. (1928). "École unique et éducation intégrale ». Revue de métaphysique et de morale, $n^{\circ} 3$, p. 409-419.

DONEGANI J.-M. \& SADOUN M. (1976). « La réforme de l'enseignement secondaire en France depuis 1945: analyse d'une non-décision ". Revue française de science politique, vol. 26, $\mathrm{n}^{\circ} 6$, p. 1125-1146.

DREYFUS F.-G. (1965). « Un groupe de pression en action, les syndicats universitaires devant le projet Billères de réforme de l'enseignement (1955-1959) ". Revue française de science politique, vol. $15, \mathrm{n}^{\circ} 2$, p. 213-250.

DuBoıs P. (2007). «Figures de l'école juste et politique scolaire dans les années fondatrices de la Troisième République ". Revue française de pédagogie : recherches en éducation, $\mathrm{n}^{\circ} 159$, p. 13-21.

FrAJERMAN L. (2007). « La FEN face aux enjeux de l'école moyenne sous la IVe République: cartographie d'un débat ". Revue française de pédagogie : recherches en éducation, $\mathrm{n}^{\circ} 159$, p. 69-79.

Garcia J.-F. (1994). L'école unique en France. Paris : PUF.

GARNier B. (2007). "Les fondateurs de l'école unique à la fin de la première guerre mondiale: I'Université nouvelle par les Compagnons ». Revue française de pédagogie : recherches en éducation, $\mathrm{n}^{\circ} 159$, p. 35-45.

Gaulle C. de (1959). Mémoires de guerre. Tome 3. Le salut (1944-1946). Paris : Plon.
ISAMBERT-JAMATI V. (1972). "Brève histoire d'une notion incertaine: la démocratisation ", Cahiers pédagogiques, $\mathrm{n}^{\circ} 107$, p. 3-7.

LACHAISE B. (1993). Yvon Delbos, 1885-1956. Périgueux: FANLAC.

Mialaret G. (1997). Le plan Langevin-Wallon. Paris : PUF, 1997.

MoLE F. (2007). « L'égalité dans la diversité : recherche d'un principe d'unification des ordres d'enseignement (18981910) ". Revue française de pédagogie: recherches en éducation, $\mathrm{n}^{\circ} 159$, p. 23-33.

Prost A. (1968). Histoire de l'enseignement en France, 18001967. Paris : A. Colin.

Prost A. (2006). "Mixité sociale et démocratisation : l'enseignement secondaire entre les deux guerres ». Communication au colloque Repenser la justice (Lyon: INRP, 15-16-17 mai 2006).

Rawls J. (1987). Théorie de la justice. Paris : Éd. du Seuil.

Robert A. D. (2004). "Jeux croisés des syndicats d'enseignants face aux réformes et projets de réforme (1944-2000) ». In J. Girault (dir.), Les enseignants dans la société française au $x x^{e}$ siècle: itinéraires, enjeux, engagements. Paris: Publications de la Sorbonne, p. $113-132$.

Robert A. D. (2005). "Les professeurs des classes élémentaires des lycées et leur représentation: crépuscule et postérité d'une idéologie catégorielle (1881-1965)". In P. Caspard, J.-N. Luc \& P. Savoie (dir.), Lycées, lycéens, lycéennes: deux siècles d'histoire. Lyon: INRP, p. 317-329.

Robert A. D. (2006a). «C. de Gaulle, l'école et l'économie ». In V. Troger (coord.) Une histoire de l'éducation et de la formation. Auxerre : Éd. Sciences humaines, p. 183-196.

Robert A. D. (2006b). "Une culture "contre" l'autre: les idées de l'éducation nouvelle solubles dans l'institution scolaire d'État? Autour de la démocratisation de l'accès au savoir ". Pædagogica Historica, vol. 42, $\mathrm{n}^{\circ} 1-2$, p. 249-261.

VINOKUR A. (2002). "Enseignement supérieur: "un changement sans réforme"? ". Formation-Emploi, $\mathrm{n}^{\circ} 79$, p. 19-30.

WALLON H. (1952). " $30^{\mathrm{e}}$ anniversaire du Groupe français d'éducation nouvelle (GFEN) ". Pour l'ère nouvelle, $\mathrm{n}^{\circ} 10$, p. 2-6.

WALzER M. (1997). Sphères de justice, une défense du pluralisme et de l'égalité. Paris : Éd. du Seuil. 